\title{
A New Journal to Improve Care for Adolescent and Young Adult Oncology Patients and Survivors
}

\author{
Leonard S. Sender, MD
}

$\mathrm{T}$ HIS IS AN EXCITING TIME for the rapidly emerging field of adolescent and young adult (AYA) oncology. The AYAO discipline arose from the growing recognition that adolescent and young adult-aged patients and survivors are not just 'old' pediatric patients or 'young' adult patients. AYAs experience cancer treatment and survivorship-related issues differently from older or younger patients and often have concerns unique to their age range. A number of research studies and reports and the views of professional organizations and individuals, governments and universities, patients and survivors, families and friends, all add up to one increasingly hard-to-ignore truth: AYA cancer patients and survivors deserve better care.

This groundswell-and the awareness of what truly creates and moves a medical specialty forward-led to the founding of Journal of Adolescent and Young Adult Oncology (JAYAO), the official journal of the newly founded Society for Adolescent and Young Adult Oncology (SAYAO).

Several factors contributed to the growing recognition that adolescents and young adults are a distinct group of cancer patients that have not enjoyed the same improvements in overall survival compared with their younger and older counterparts. The reasons for the differences have not been fully elucidated, but research increasingly points to multiple factors, including biological differences, treatment protocol or medical care facility variances, lack of relevant clinical trials, and restrictions in access to care due to uninsured or underinsured status.

AYA cancer presents the medical community with several unique challenges. It requires true collaboration between pediatric and medical oncologists as the age range crosses both disciplines. Our AYA cancer patients not only have cancer but are often dealing with ongoing developmental and psychosocial issues at the same time; as such, we must be aware of how a cancer diagnosis interferes with their normal development. In addition to newly diagnosed AYA-aged patients, pediatric cancer survivors who have 'aged in' to AYAO are a growing part of the community. Cancer is increasingly viewed as a chronic disease and survivorship is often fraught with the unintended consequences of cancer treatment, including infertility, heart and lung damage, and metabolic problems.

JAYAO will have a very broad mandate. Dedicated to the promotion of multidisciplinary, clinical translational research, best clinical practices, education, communication, and collaboration between health professionals in AYA oncology,
JAYAO will meet the urgent need to foster understanding of and improve cancer care and outcomes for AYA-aged cancer patients and survivors. JAYAO will be the central forum and authoritative source for the full spectrum of AYA oncology, including clinical, epidemiological, psychosocial, survivorship, and other AYA oncology-focused topics.

JAYAO will loosely focus on the AYAO age range determined by the National Cancer Institute here in the United States-15 to 39. I say loosely because it is just that-some articles may skew slightly younger or older, and many may focus on only a subset of this age range.

As you can see from this inaugural issue, we have already been successful in assembling a wide range of provocative articles and perspectives that are extremely timely and relevant to the field of AYA oncology. In this first issue, you will find Review articles, Original articles, a Roundtable discussion with leaders in the field, a Controversies feature of responses to a timely AYAO issue, articles from leading Advocacy groups about their organization or a particular program within it, a Clinical Trial Spotlight highlighting a trial with AYA-specific impacts, and a Point of View from pediatric practitioners. Voices of AYAO stories from survivors provide illumination about these patients' experiences and challenges. Future $J A Y A O$ issues will have Case Studies with AYA-impact enhancement and a Perspectives feature with viewpoints from both a pediatric and an adult practitioner on a topic related to AYA patients and survivors. JAYAO will be highly competitive and all articles will be rigorously peer-reviewed.

$J A Y A O$ is an interdisciplinary journal, not only because the issues in AYAO are grounded in basic, clinical, and psychosocial research and services, but because true change in a field is best effected through transdisciplinary collaboration and awareness. Any professional working with AYA cancer patients on any level should find something of relevance in JAYAO. Our readership includes but is not limited to: pediatric, medical, and surgical oncologists of all types and specialties; oncology nurses and advanced practice staff; psychosocial and supportive care providers including psychiatrists, psychologists, and social workers; translational cancer researchers; and academic and community-based pediatric and adult cancer institutions.

We are fortunate to have assembled an outstanding multidisciplinary international editorial board, including prominent associate editors and section editors representing the AYAO-relevant subspecialties of breast cancer, gastrointestinal 
oncology, gynecologic oncology, head and neck cancer, leukemia and lymphoma, melanoma, neuro-oncology, oncofertility, radiation oncology, testicular cancer, sarcoma, and psychosocial and survivorship issues. Many of you have already met or interacted with our Managing Editor, Rebecca Morris, who has a strong commitment to the field and is a central resource for authors submitting to the Journal.

There are a lot of catchphrases in AYAO; "Mind the Gap" is perhaps the most familiar one. I like to use a different one"splitters and lumpers." So much excellent groundwork has been laid in establishing the need for AYAO as a distinct area of cancer focus, but this has often been accomplished by "lumping" those in the 15 to 39 age range together. I hope that $J A Y A O$ enables our field to increasingly conduct "splitting" research, really digging down deep within each issue and disease to focus on teasing out the jewels that will lead to significant improvements in the cancer treatment and survivorship experiences of young people in this age range.

Significant advances in AYAO will need to be driven by our professional collaboration and interaction. We hope that each of you, whether or not AYAs are the majority or minority of your professional focus, will truly embrace and engage with
JAYAO by submitting articles, participating in responsebased features such as Controversies, and signing up and participating as a reviewer.

To truly advance care for this population, AYA oncology must be recognized as a distinct subspecialty, with a professional organization and publication to support it. JAYAO is the culmination of this impetus, and a major new professional organization dedicated to excellence in this field-the Society for Adolescent and Young Adult Oncology (SAYAO)-will be a key partner in moving our field forward.

Collectively, we have the ability to transform cancer care and survivorship for AYAs, and I invite you to join this movement as an active participant in this new Journal and resource, truly a monumental step in improving care for the underserved AYA oncology population. Our hope is that $J A Y A O$ will bring the field of AYAO to a whole new level to improve patient and survivorship care and outcomes. Together, we move forward. 